\title{
Stable isotope labeled metabolomics improves identification of novel metabolites and pathways
}

"The recent surge in bioanalytical and bioinformatics methods developed for metabolomics studies have significantly improved our ability to expand isotope-labeling studies ... bringing us closer to the promise of a true systems biology understanding of the metabolism for many organisms."

Keywords: carbon-13 $\approx$ fluxomics $\approx$ metabolic pathway $\approx$ metabolite identification $\approx$ metabolomics $\approx$ stable isotope labelling

Metabolomics is a rapidly growing field of postgenomic biology that enables systemwide studies of cellular metabolism. Stable isotope labeling is an established approach for investigation of the dynamics of metabolic pathways. The combination of these two approaches promises to provide a comprehensive understanding of metabolic networks in biological systems.

\section{Metabolomics}

Metabolomics involves the semi-quantitative detection of many small molecules $(<1500 \mathrm{Da})$ in a biological system using modern bioanalytical platforms such as NMR, GC-MS, CE-MS and LC-MS. Metabolomics studies are generally classified as targeted or untargeted. Targeted studies involve quantitative analysis of a subset of metabolites or metabolic pathways with high accuracy and confidence. On the other hand, untargeted studies analyze all detectable metabolite signals from a chosen analytical platform, or a combination of platforms, for enhanced coverage of the metabolome. Untargeted studies are generally semiquantitative in nature, and offer the advantage of providing an unbiased analysis, with the potential to discover novel metabolites or metabolic pathways [1].

The major limitation associated with untargeted metabolomics is the identification of metabolites [1]. Recent advances in highresolution (HR)MS with ESI have significantly improved our ability to assign a molecular formula to each detected molecular ion, based on accurate mass and natural isotope abundance [2]. However, secondary analytical approaches are usually required for confident identification, which often requires the purchase, synthesis or isolation of pure authentic standards [1]. Another major limitation of untargeted metabolomics is the lack of dynamic information to allow mechanistic interpretation of data in the context of metabolic pathways [3-6]. While untargeted metabolomics may demonstrate an increased abundance of a certain metabolite under test conditions, it cannot show whether this is a result of increased flux in a synthetic enzyme, decreased flux in a consuming enzyme, or alteration in transport of the metabolite into or out of the cell. Furthermore, many metabolic pathways are interconnected, and metabolite levels are often maintained by a number of metabolic processes, which cannot be adequately delineated by measurement of metabolite concentrations alone. For example, glutamate can participate in up to 200 metabolic reactions [4].

\section{Metabolite identification with stable isotopes}

Stable isotope labeling provides vast improvements in both metabolite identification and pathway characterization. Metabolite identification in HRMS metabolomics is achieved by determination of the molecular formula for each putative metabolite. Improved confidence in molecular formula assignment can be achieved in cell-culture studies where cells can be grown on a uniformly ${ }^{12} \mathrm{C}$ - (unlabeled) or uniformly ${ }^{13} \mathrm{C}$-labeled carbon source [7]. In this case, the mass difference between labeled and unlabeled metabolites clearly defines the number of carbon atoms in each molecule, which significantly reduces the search space for identification of

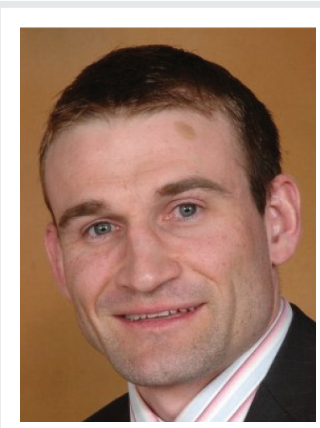

Darren J Creek

Department of Biochemistry \& Molecular Biology, Bio2I Molecular Science \& Biotechnology Institute, University of Melbourne,

Flemington Rd, Parkville, VIC 30I0, Australia

E-mail: darrencreek@gmail.com 
possible molecular formulas with an accurate mass that agrees with the detected mass [8]. Even with the correct molecular formula it is often difficult to accurately identify metabolites where a number of possible isomers exist. However, in organisms with known metabolic pathways it is sometimes possible to identify isomeric metabolites based on the isotope labeling patterns from specific nutrients. For example, $\mathrm{C}_{6} \mathrm{H}_{6} \mathrm{O}_{6}$ is likely to be cis-aconitate if the $\mathrm{U}-{ }^{13} \mathrm{C}$-glucose-labeled isotopomer abundances resemble the citrate labeling pattern (examples in [4] and [9]), but is more likely to represent dehydroascorbate if the metabolite is unlabeled in cells auxotrophic for ascorbate (or if fully labeled in ascorbate-producing cells).

\section{Metabolic pathway elucidation}

The major advantages of incorporating stable isotope labeling with metabolomics are in the understanding of flux in known pathways and the identification of novel pathways. Incorporation of labeled nutrients allows immediate differentiation of endogenous metabolites synthesized by the cells from the exogenous compounds taken up from the growth medium. This allows a vast simplification of metabolomics data when attempting to study intracellular endogenous metabolism, and confirms the biosynthetic nature of metabolites detected in untargeted studies [7].

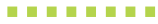 \\ "The major advantages of incorporating stable isotope labeling with metabolomics are in the understanding of flux in known pathways and the identification of novel pathways."}

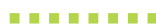

The placement of novel metabolites in the context of metabolic pathways can be achieved by performing metabolomics analysis with a mixture of labeled and unlabeled nutrients (e.g., 50\% $\mathrm{U}-{ }^{13} \mathrm{C}$-glucose [10]) or with partly labeled nutrients (e.g., 1,2-13 C-glucose [11]). These approaches allow the determination of biosynthetic routes for each metabolite by interpretation of isotopomer patterns in the context of possible metabolic pathways $[10,11]$. Recent examples of elucidation of novel pathways by isotope labeling include the discovery of riboneogenesis in yeast [12], and the GABA shunt in Toxoplasma [9].

Detailed pathway elucidation often requires the collection of time-course data to follow incorporation of label into pathway intermediates $[5,6]$. These studies ideally require a rapid addition or replacement of growth medium with labeled nutrient, and collection of samples for isotopomer analysis at subsequent time intervals. An alternative approach is the application of chemostat culture conditions where unlabeled nutrient is replaced with labeled nutrient by constant infusion [5]. While data interpretation from this method is mathematically more complex, it avoids potential stress-mediated metabolic perturbations in response to the manipulation required for medium replacement in a batch culture system.

Isotope-labeling studies ideally require analysis of all possible isotopomers for each metabolite, and are commonly limited to investigation of a targeted set of metabolites in order to minimize the overwhelming task of data analysis. However, with recent advances in HR LC-MS and bioinformatics software, it is now possible to study isotope labeling across the entire detected metabolome $[10,13]$. Modern mass analyzers with resolutions greater than 40,000 provide sufficient isotopic resolution for most small molecules to allow untargeted detection of all isotopomers with minimal background noise. Therefore, evaluation of isotopomer distributions can be performed by automated extraction of mass signals corresponding to multiples of the isotopic mass difference (i.e., $\Delta \mathrm{m}=1.00335 / z$ for ${ }^{13} \mathrm{C}$ ), within the mass accuracy window of the spectrometer (ideally $<2$ ppm) $[2,13]$. Further selectivity is achieved by incorporation of chromatographic information due to the fact that isotopomers are expected to co-elute (with the exception of deuterated compounds). Algorithms that detect peaks with correlated retention times and peak shapes offer improved detection of putative isotopomers [14,15]. A number of bioinformatics tools have recently been developed to automate the process of isotope detection for metabolomics data, including mzMatch.R [13,14], CAMERA [15], MetExtract [8] and MAVEN [16]. While automated detection software is essential for metabolomewide isotope analysis, it is generally expected that significant peaks are manually inspected, and controls from unlabeled cells and labeled growth medium are analyzed, to remove false-positives that arise from background signals.

\section{Other roles for stable isotope labeling in metabolomics}

The role of stable isotopes in metabolomics extends beyond the discovery of novel metabolites and pathways. Stable isotope labeling combined with quantitative metabolite analysis allows 
determination of flux through metabolic pathways [3-5]. Mathematical modeling of flux data from whole-cell experiments potentially enables predictive modeling of metabolism on a comprehensive systems scale. However, the requirement for concurrent absolute quantification limits these analyses to a subset of selected metabolites that can be accurately quantified with appropriate calibration standards and IS.

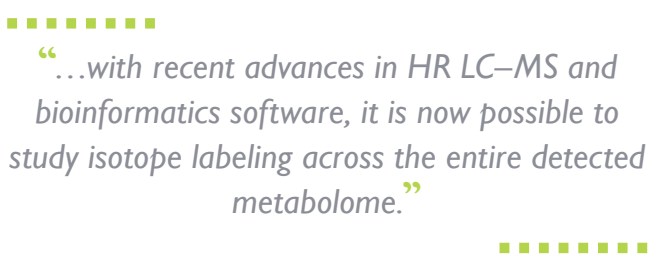

The scope of metabolites that can be accurately quantified is increasing as methods are becoming available for quantification of hundreds of metabolites with multiple IS [17]. Complete stable isotope labeling of cell culture is a convenient method to obtain multiple IS of relevant metabolites for quantitative studies [18].

An additional role of stable isotope labeling is the surrogate detection of metabolic pathways in cases where individual metabolites cannot be directly measured. Missing metabolites in pathways are common in metabolomics studies due to the wide dynamic range of intracellular metabolite concentrations, and the inability of a single analytical method to detect metabolites with vastly different physicochemical properties. Measurement of stable isotope incorporation into metabolic end-products can provide inference of the presence of metabolic intermediates in predicted metabolic pathways. This concept is employed in fluxomics approaches such as Fiatflux [19] and SIDMAP [20] to simplify flux analysis of central metabolic pathways by isotopomer analysis of defined pathway end products.

\section{Future perspective}

It is expected that stable isotope labeling will become common practice in metabolomics studies in the near future. All the necessary tools are accessible, with widespread application of high resolution MS-based metabolomics and recent bioinformatics advances for automated isotopomer detection[10]. Numerous stable isotope-labeled nutrients are readily available that allow comprehensive analysis of most aspects of cellular metabolism, although the cost of these labeled pre-cursors may prohibit extensive studies, particularly for in vivo mammalian systems. Nevertheless, there are already examples of ${ }^{13} \mathrm{C}$-labeled metabolic profiling in whole animal and human models [4].

The recent surge in bioanalytical and bioinformatics methods developed for metabolomics studies have significantly improved our ability to expand isotope-labeling studies to the whole-systems level, bringing us closer to the promise of a true systems biology understanding of the metabolism for many organisms. It is hoped that continued advances in bioinformatics will optimize the workflow from detection of labeled metabolites to updating annotations and interpretation of flux for novel metabolites and pathways.

\section{Financial \& competing interests disclosure}

D Creek is supported by an Australian National Health and Medical Research Council training fellowship. The author has no other relevant affiliations or financial involvement with any organization or entity with a financial interest in or financial conflict with the subject matter or materials discussed in the manuscript apart from those disclosed.

No writing assistance was utilized in the production of this manuscript.

\section{References}

1 Dunn WB, Erban A, Weber RJM et al. Mass appeal: metabolite identification in mass spectrometry-focused untargeted metabolomics. Metabolomics 9(1), 44-66 (2013).

2 Creek DJ, Jankevics A, Burgess KEV, Breitling R, Barrett MP. IDEOM: an Excel interface for analysis of LC-MS-based metabolomics data. Bioinformatics 28(7), 1048-1049 (2012).

3 Klein S, Heinzle E. Isotope labeling experiments in metabolomics and fluxomics. WIREs Syst. Biol. Med. 4(3), 261-272 (2012).
4 Fan TWM, Lorkiewicz PK, Sellers K, Moseley HNB, Higashi RM, Lane AN. Stable isotope-resolved metabolomics and applications for drug development. Pharmacol. Ther. 133(3), 366-391 (2012).

5 Winder CL, Dunn WB, Goodacre R. TARDIS-based microbial metabolomics: time and relative differences in systems. Trends Microbiol. 19(7), 315-322 (2011).

6 Zamboni N, Sauer U. Novel biological insights through metabolomics and ${ }^{13} \mathrm{C}$-flux analysis. Curr. Opin. Microbiol. 12(5), 553-558 (2009).
7 Giavalisco P, Köhl K, Hummel J, Seiwert B, Willmitzer L. ${ }^{13} \mathrm{C}$ isotope-labeled metabolomes allowing for improved compound annotation and relative quantification in liquid chromatographymass spectrometry-based metabolomic research. Anal. Chem. 81(15), 6546-6551 (2009).

8 Bueschl C, Kluger B, Berthiller F et al. MetExtract: a new software tool for the automated comprehensive extraction of metabolite-derived LC/MS signals in metabolomics research. Bioinformatics 28(5), 736-738 (2012). 
9 Macrae JI, Sheiner L, Nahid A, Tonkin C, Striepen B, McConville MJ. Mitochondrial metabolism of glucose and glutamine is required for intracellular growth of Toxoplasma gondii. Cell Host Microbe 12(5), 682-692 (2012).

10 Creek DJ, Chokkathukalam A, Jankevics A, Burgess KEV, Breitling R, Barrett MP. Stable isotope-assisted metabolomics for networkwide metabolic pathway elucidation. Anal. Chem. 84(20), 8442-8447 (2012).

11 Metallo CM, Walther JL, Stephanopoulos G. Evaluation of ${ }^{13} \mathrm{C}$ isotopic tracers for metabolic flux analysis in mammalian cells. J. Biotechnol. 144(3), 167-174 (2009).

12 Clasquin MF, Melamud E, Singer A et al. Riboneogenesis in yeast. Cell 145(6), 969-980 (2011).

13 Chokkathukalam A, Jankevics A, Creek DJ, Achcar F, Barrett MP, Breitling R.
mzMatch-ISO: an R tool for the annotation and relative quantification of isotope-labeled mass spectrometry data. Bioinformatics 29(2), 281-283 (2013).

14 Scheltema RA, Jankevics A, Jansen RC, Swertz MA, Breitling R. PeakML/mzMatch: A file format, java library, R library, and tool-chain for mass spectrometry data analysis. Anal. Chem. 83(7), 2786-2793 (2011).

15 Kuhl C, Tautenhahn R, Böttcher C, Larson TR, Neumann S. CAMERA: an integrated strategy for compound spectra extraction and annotation of liquid chromatography/ mass spectrometry data sets. Anal. Chem. 84(1), 283-289 (2011).

16 Melamud E, Vastag L, Rabinowitz JD. Metabolomic analysis and visualization engine for LCMS data. Anal. Chem. 82(23), 9818-9826 (2010).
17 Griffiths WJ, Koal T, Wang Y, Kohl M, Enot DP, Deigner H-P. Targeted metabolomics for biomarker discovery. Angew. Chem. Int. Ed. Engl. 49(32), 5426-5445 (2010).

18 Wu L, Mashego MR, Van Dam JC et al. Quantitative analysis of the microbial metabolome by isotope dilution mass spectrometry using uniformly ${ }^{13} \mathrm{C}$-labeled cell extracts as internal standards. Anal. Biochem. 336(2), 164-171 (2005).

19 Zamboni N, Fischer E, Sauer U. FiatFlux a software for metabolic flux analysis from ${ }^{13} \mathrm{C}$-glucose experiments. BMC Bioinf. 6(1), 209 (2005).

20 Boros LG, Brackett DJ, Harrigan GG. Metabolic biomarker and kinase drug target discovery in cancer using stable isotope-based dynamic metabolic profiling (SIDMAP). Curr. Cancer Drug Targets 3(6), 445-453 (2003). 